\title{
Colopexia laparoscópica com retalho de tela de polipropileno em cães
}

\author{
[Laparoscopic colopexy with polyprolyne mesh in dogs] \\ M.V. Brun ${ }^{1}$, L.D. Guimarães ${ }^{1}$, H.H.A. Barcellos ${ }^{1}$, N. Guizzo Júnior ${ }^{2}$, R.A. Pereira ${ }^{1}$ \\ ${ }^{1}$ Faculdade de Agronomia e Medicina Veterinária-UPF \\ Caixa Postal 611 \\ 99001-970 - Passo Fundo, RS \\ ${ }^{2}$ Aluno de graduação - FAMV-UPF - Passo Fundo, RS
}

\begin{abstract}
RESUMO
Avaliou-se uma nova técnica de colopexia laparoscópica, sem necessidade de suturas intracorpóreas em cães, utilizando a implantação de segmento de tela de polipropileno em 11 animais. As superfícies serosas do cólon descendente e parede muscular foram mantidas em contato com o implante posicionado por meio de lesão produzida no mesocólon e sob o retalho bipediculado de músculo transverso abdominal. As extremidades da tela foram unidas com a aplicação de clipe de titânio. Os procedimentos duraram $36,00 \pm 13,15 \mathrm{~min}$, sem a ocorrência de complicações. No período pós-operatório, foi verificada a manutenção da colopexia em sete animais, a formação de fixação parcial entre o intestino e a parede em três cães, e a não formação de aderências do cólon em um paciente. Conclui-se que o procedimento proposto é viável para cães, porém necessita de ajustes técnicos.
\end{abstract}

Palavras-chave: cão, videocirurgia, endocirurgia, videolaparoscopia

\begin{abstract}
A new laparoscopic colopexy technique was tested in dogs without intracorporeal sutures. The use of polypropylene mesh in eleven mongrel dogs was evaluated. The serosal surface of the descendent colon and transverse abdominal muscle were put in contact with the mesh application, taking into consideration that the polypropylene implant was positioned through the mesocolon and under the muscle flap. Finally, the extremities of the mesh were clipped. The procedures lasted 36:00 $\pm 13: 15 \mathrm{~min}$, without any kind of complications. In the post-operative period, adequate colopexy in seven dogs was observed. In three dogs the colopexy was partial and in one the bowel did not adhere to the muscles or mesh. The proposed procedure is viable for dogs, however, it is necessary to make some adjustments.
\end{abstract}

Keywords: dog, videsurgery, endosurgery, videolaparoscopy

\section{INTRODUÇÃO}

Considerando a importância que a colopexia apresenta no manejo do prolapso recidivante em cães (Fossum et al., 1994; Fossum et al., 2002), alguns autores desenvolveram diferentes técnicas laparoscópicas ou vídeo-assistidas, envolvendo ou não a realização de suturas intracorpóreas (Brun et al., 2004c,d; Thompson e Hendrickson, 1998). Freeman (1998) e Thompson e
Hendrickson (1998) descreveram a possibilidade de execução da colopexia laparoscópica, contudo não detalharam profundamente sobre os aspectos técnicos. Os primeiros autores citaram que devem ser empregados três portais, um na linha média ventral e outros dois no quadrante abdominal caudal direito, e que a camada seromuscular do cólon deve ser fixada à parede muscular em padrão interrompido ou contínuo simples. Os outros autores sugeriram que é

Recebido em 10 de agosto de 2005

Aceito em 22 de novembro de 2006

E-mail: mbrun@upf.br 
necessário posicionar os trocartes nos quadrantes abdominais caudais e realizar suturas inabsorvíveis ao longo do cólon descendente.

Brun et al. (2004c,d) desenvolveram duas diferentes técnicas endoscópicas, uma preconizando a realização de suturas intracorpóreas e a outra a partir de pontos transparietais. No procedimento incisional laparoscópico, esses autores descreveram o emprego de quatro portais, um localizado na linha média ventral $(10 \mathrm{~mm})$, dois na parede abdominal direita $(5 \mathrm{~mm}$ e $10 \mathrm{~mm})$ e um na parede esquerda $(5 \mathrm{~mm})$. Após a aproximação do cólon à musculatura abdominal com pinça Babcock, realizaram-se incisões na superfície antimesentérica do cólon (nas camadas serosa e muscular) e na parede esquerda, que foram, então, unidas com duas suturas intracorpóreas contínuas simples. Os autores demonstraram que o procedimento incisional esteve associado à formação de aderências permanentes em todos os animais com características histológicas e resistência à tração similares às obtidas pela técnica incisional por celiotomia. As observações experimentais obtidas a partir de diferentes estudos (Brun et al., 2004d,g) permitiram a aplicação da técnica incisional laparoscópica em cães com prolapso retal recidivante (Brun et al., 2004b,e,f). Todos os pacientes operados evoluíram sem a ocorrência de complicações ou recidivas.

Brun et al. (2004c) desenvolveram uma técnica vídeo-assistida com o intuito de minimizar o número de portais, bem como o tempo operatório, além de isentar a necessidade de suturas intracorpóreas. Na colopexia com sutura transparietal, os autores aplicavam a sutura através da pele e acompanhavam, por visibilização endoscópica, sua introdução na superfície antimesentérica do cólon. Apesar de factível e de demonstrar a formação de aderências permanentes, tal técnica tornou-se contra-indicada para cães, haja vista a formação de lesões dérmicas e/ou nos planos mais profundos nos locais de contato com os materiais utilizados para o apoio das suturas.

Rawllings et al. (2002) descreveram procedimento para a fixação do cólon associado a poucas dificuldades técnicas. Para tanto, são necessários apenas dois portais, sendo que, através do posicionado na parede esquerda, foi introduzida pinça Babcock, o que permitiu a apreensão e o tracionamento do intestino através da parede abdominal, a partir da ampliação da ferida muscular de acesso. O cólon foi, então, suturado à parede muscular pelo acesso aberto.

Mais recentemente, foram testadas membranas conservadas em solução hipersaturada de sal a $75 \%$ (Brun et al., 2004a) ou glicerina ${ }^{*}$ em colopexias, com o intuito de isentar a necessidade das suturas intracorpóraes e de aplicação das suturas transparietais, contudo as fixações não foram adequadamente mantidas em nenhum paciente, possivelmente pela degradação dos implantes junto ao flapes musculares sobre os quais eram posicionados. Essas experimentações permitiram observar que a seqüência técnica proposta poderia se tornar apropriada na obtenção de aderência permanente do cólon, desde que o material implantado fosse de outra natureza. Assim, no presente trabalho, objetivou-se avaliar o emprego de tela de polipropileno na colopexia de cães, considerando seus aspectos técnicos e resultados trans e pósoperatórios.

\section{MATERIAL E MÉTODOS}

Foram utilizadas 11 cadelas adultas hígidas, com peso médio de $6,15 \pm 1,45 \mathrm{~kg}$. Após o jejum sólido de $12 \mathrm{~h}$ e líquido de duas horas, as pacientes foram submetidas à tricotomia das paredes abdominais laterais e ventral. Como pré-medicação, receberam a associação de sulfato de morfina ${ }^{1}\left(0,3 \mathrm{mg} \cdot \mathrm{kg}^{-1}\right.$, IM) e maleato de acetilpromazina ${ }^{2}\left(0,1 \mathrm{mg} \cdot \mathrm{kg}^{-1}, \mathrm{IM}\right)$ na mesma seringa hipodérmica. $\mathrm{Na}$ seqüência, foram induzidas com propofol ${ }^{3}\left(4-11,2 \mathrm{mg} \cdot \mathrm{kg}^{-1}, \mathrm{IV}\right)$ e mantidas sob anestesia geral inalatória com halotano a 1,5 a $2 \mathrm{~V} \%$, vaporizado em $\mathrm{O}_{2}$, a $100 \%$ em circuito semi-fechado. Trinta minutos antes dos procedimentos, administrou-se ampicilina sódica ${ }^{5}$ (20mg. $\mathrm{kg}^{-1}$, IV). Durante toda a operação, os pacientes foram hidratados com solução de Ringer lactato ${ }^{1}\left(10 \mathrm{ml} \cdot \mathrm{kg}^{-1}\right.$ a cada hora).

Com os animais em decúbito dorsal, promoveuse incisão pós-umbilical na linha média ventral,

\footnotetext{
* BRUN (comunicação pessoal) - UPF-RS, 2006.

${ }^{1}$ Dimorf, Cristália, Itapira, SP.

2 Acepran 1\%.; Univet, SP, SP.

${ }^{3}$ Diprivan, Cristália, Itapira, SP.

${ }^{4}$ Tanohalo, Cristália, Itapera, SP.

${ }^{5}$ Ampicilina sódica 1g, Teuto, Anápolis, GO.
} 
aproximadamente $1 \mathrm{~cm}$ caudal à cicatriz umbilical. Os folhetos externos do músculo reto abdominal foram apreendidos com duas pinças Halsted, permitindo o acesso à cavidade sob visibilização direta. Introduziu-se por essa ferida um trocarte ${ }^{6} \mathrm{de} 10 \mathrm{~mm}$, que permitiu a insuflação da cavidade com $\mathrm{CO}_{2}$ medicinal até alcançar a pressão de $12 \mathrm{mmHg}$, na velocidade de $2 \mathrm{~L} \cdot \mathrm{min}^{-1}$.

A cavidade foi inspecionada com endoscópio rígido $^{7}$ de $10 \mathrm{~mm}$ e $0^{0}$, acoplado à microcâmera ${ }^{8} \mathrm{e}$ fonte de luz halógena ${ }^{9}$. Outros dois trocartes $(10 \mathrm{~mm} \text { e } 5 \mathrm{~mm})^{10}$ foram posicionados nas paredes abdominais direita e esquerda, caudalmente ao primeiro e em ângulo aproximado de $45^{\circ}$ em relação a este. O cólon descendente foi apreendido com pinça Babcock ${ }^{11}$ e tracionado crânio-lateralmente até a região proposta para a colopexia.

Junto ao segmento de intestino, promoveu-se breve divulsão do mesocólon com pinça Maryland $^{12}$. Na seqüência, foi realizada, na região eleita para colopexia, uma incisão transversal de aproximadamente $2 \mathrm{~cm}$ no músculo transverso abdominal esquerdo com tesoura de Metzenbaum $^{13}$. Outra incisão muscular de dimensões semelhantes foi promovida paralelamente, permitindo a produção de flap muscular bipediculado a partir da dissecação do tecido entre os músculos transverso e oblíquo abdominal interno. Introduziu-se no abdome um retalho de tela de polipropileno de $12 \times 1,5 \mathrm{~cm}$ através de redutor ${ }^{14}$, sendo uma das extremidades do implante transpassada através da ferida produzida no mesocólon, posicionada sobre o flap muscular, e mantida aproximada da outra com uma pinça de apreensão ${ }^{15}$. As duas extremidades do retalho foram unidas com a aplicação de um clipe de titânio $^{16}$ abaixo da pinça (Fig. 1), tendo-se o cuidado de não

\footnotetext{
6 Trocarte $10 \mathrm{~mm}$ 141201, Edlo, Canoas, RS

${ }^{7}$ Endoscópio rígido Hoopkins $5 \mathrm{~mm} 30^{\circ}, \mathrm{H}$. Strattner, Rio de Janeiro, RJ.

8 Toshiba CCD, China.

${ }^{9}$ Fonte de luz fria halógena, H. Strattner, Rio de Janeiro, RJ.

10 Trocarte 5mm 141208, Edlo, Canoas, RS.

11 Pinça Babcock, Ethicon, São José dos Campos, SP.

12 Pinça Maryland 141476, Edlo, Canoas, RS.

13 Tesoura de Metzenbaum 141439, Edlo, Canoas, RS.

14 Redutor tipo diafragma 141060 Edlo, Canoas, RS.

15 Pinça de aprrensão 140976, Edlo, Canoas, RS.

16 Atrauclip, Weck Closure Systems, U.K.
}

comprimir demasiadamente as estruturas envolvidas, a partir da manutenção de espaço entre a tela e o cólon que permitia a passagem da pinça de Maryland. $\mathrm{O}$ excedente de tela sobre o clipe foi seccionado com tesoura ${ }^{17}$ e removido do abdome pelo redutor.

A cavidade foi parcialmente desinsuflada até alcançar a pressão de $5 \mathrm{mmHg}$ e, na ausência de hemorragias, drenou-se o restante do $\mathrm{CO}_{2}$, e os três portais foram removidos sob visibilização direta. As feridas maiores foram suturadas em dois planos, o primeiro em nível muscular em padrão de Sultan com náilon monofilamentar $3-0^{18}$, e o segundo envolvendo a pele em padrão interrompido simples. As feridas menores foram ocluídas apenas no nível da pele. A analgesia foi providenciada com a aplicação de Ketoprofeno ${ }^{19}$ (2mg.kg ${ }^{-1}, \mathrm{SC}, \mathrm{SID}, 3 \mathrm{~d}$.), e as feridas operatórias foram diariamente higienizadas com solução de $\mathrm{NaCl}$ 0,9\% (TID, 7d).

Todos os animais foram submetidos às laparoscopias pós-operatórias, em períodos que variaram de 25 a 64 dias, a citar (em parênteses, consta o número de pacientes operados de acordo com o período): 25(1), 29(1), 30(4), 35(1), 38(1), 42(2) e 64(1). Nesse procedimento, foram utilizados três portais dispostos de forma semelhante à previamente descrita. Coletou-se amostra envolvendo a tela e os tecidos adjacentes nos pacientes avaliados ao trigésimo e quadragésimo segundo dias dos pós-operatórios. Nesse exame considerou-se a ocorrência de fibrose, células inflamatórias mononucleares e neovascularização, sendo atribuídos a cada uma dessas características valores que poderiam variar de 0 a 3, que correspondiam: 0 ausência da característica; 1 pequena presença; 2 moderada ocorrência; e 3 acentuada evidência. Na avaliação do procedimento proposto, consideraram-se a viabilidade técnica, as dificuldades operatórias, o tempo cirúrgico, as complicações trans e pósoperatórias, a manutenção da colopexia, a formação de outras aderências intra-abdominais e as características histológicas das biopsias.

\footnotetext{
17 Tesoura tipo gancho 190987, Edlo, Canoas, RS.

18 Mononylon 3-0 Ethilon, Ethicon- Johnson \& Johnson, São José dos Campos, SP.

${ }^{19}$ Ketofen 1\%, Rhodis-Mérieux, Paulínia, SP.
} 


\section{RESULTADOS}

Os procedimentos foram realizados em tempo médio de $36,00 \pm 13,15 \mathrm{~min}$, sem a ocorrência de complicações trans ou pós-operatórias. A técnica proposta possibilitou a fixação do cólon sem maiores dificuldades. Também não foram evidenciadas complicações durante as avaliações laparoscópicas (Fig. 2), ocasiões nas quais se verificou a manutenção da colopexia conforme obtido no primeiro procedimento em sete dos 11 pacientes $(63,6 \%)$. Três cadelas $(27,3 \%)$ apresentaram colopexia parcial, estando esse órgão afastado da parede muscular esquerda, contudo aderido a esta por meio da tela que se encontrava fixada apenas na superfície mesentérica. Um animal (9,1\%) apresentou ausência de aderências do intestino grosso com o implante e com a parede muscular, estando o órgão envolto pelo segmento de tela e com possibilidade de deslocamento cranial ou caudal com o auxílio da pinça Babcock. Em todos os animais, a tela caracterizava-se macroscopicamente recoberta por tecido conjuntivo, podendo ser prontamente reconhecida durante a avaliação da cavidade. Não houve o desenvolvimento de megacólon ou impactação fecal cranialmente ao sítio de fixação.

Dentre as aderências intra-abdominais, destacouse a fixação do omento, que ocorreu em nove dos 11 pacientes $(81,8 \%)$. Foram constatadas aderências do mesométrio em três cães e da parede vesical em um paciente. Em um animal, observaram-se, ainda, fixações do omento em outros locais (intestino delgado e linha média), bem como noutro cão do ligamento lateral da bexiga e do omento, e em outro animal do ligamento redondo do útero. Seis animais demonstraram simultaneamente mais de um tipo de aderência, sem, entretanto, configurar importância clínica. Nos exames histológicos, evidenciou-se, em três cães, que, no $30^{\circ}$ dia de pós-operatório, a tela apresentava-se envolvida por grande quantidade de tecido fibroso (grau 3). No quarto animal, o envolvimento foi moderado. Quanto às células mononucleares, os pacientes apresentaram moderada ou acentuada ocorrência dessa característica, sendo tais condições distribuídas uniformemente entre estes. Quanto à vascularização, apenas um dos cães demonstrou moderada presença dessa característica, sendo que, em todos os demais, a neovascularização era pouco evidente. Em apenas um dos pacientes avaliados nesse período, evidenciou-se a presença de gigantócitos. Nos dois cães, no dia 42, tanto a fibrose quanto a presença de células mononucleares estavam acentuadas, enquanto a vascularização estava moderadamente presente.

\section{DISCUSSÃO}

Dentre as técnicas de colopexias laparoscópicas disponíveis na literatura que isentam a necessidade de suturas intracorpóreas e que podem ser realizadas em tempo curto, tanto a operação com sutura transparietal (Brun et al., 2004c) como a com implante de pericárdio homólogo (Brun et al., 2004a) ou heterólogo* mostraram-se inapropriadas para cães, tanto pelas lesões parietais e cutâneas associada à primeira quanto pela impossibilidade de manutenção da colopexia no segundo procedimento. Considerando que apenas um animal não apresentou nenhum tipo de fixação do cólon à parede muscular, a operação proposta, se submetida a alguns pequenos ajustes técnicos, poderá ser considerada viável para cães.

Em três dos quatro animais em que o intestino não se manteve fixado diretamente ao músculo, considera-se que a tela tenha se deslocado a partir da superfície antimesentérica do cólon, já que a mesma apresentava-se aderida na superfície oposta. Pela distância observada entre tais estruturas, considera-se também que a colopexia seria inefetiva para evitar prolapso retal em paciente doente. Acredita-se que o deslocamento da tela tenha ocorrido através do único clipe de titânio aplicado, possivelmente pelo fato de esse material não abranger apropriadamente a largura completa das extremidades do implante. Deve-se considerar também que o órgão submetido à fixação manteve os movimentos peristálticos fisiológicos no pós-operatório, o que pode ter contribuído para o deslocamento da tela. Assim, os autores do presente estudo sugerem que, para próximos procedimentos dessa natureza, sejam, então, aplicados dois clipes. 


\section{Colopexia laparoscópica com retalho...}
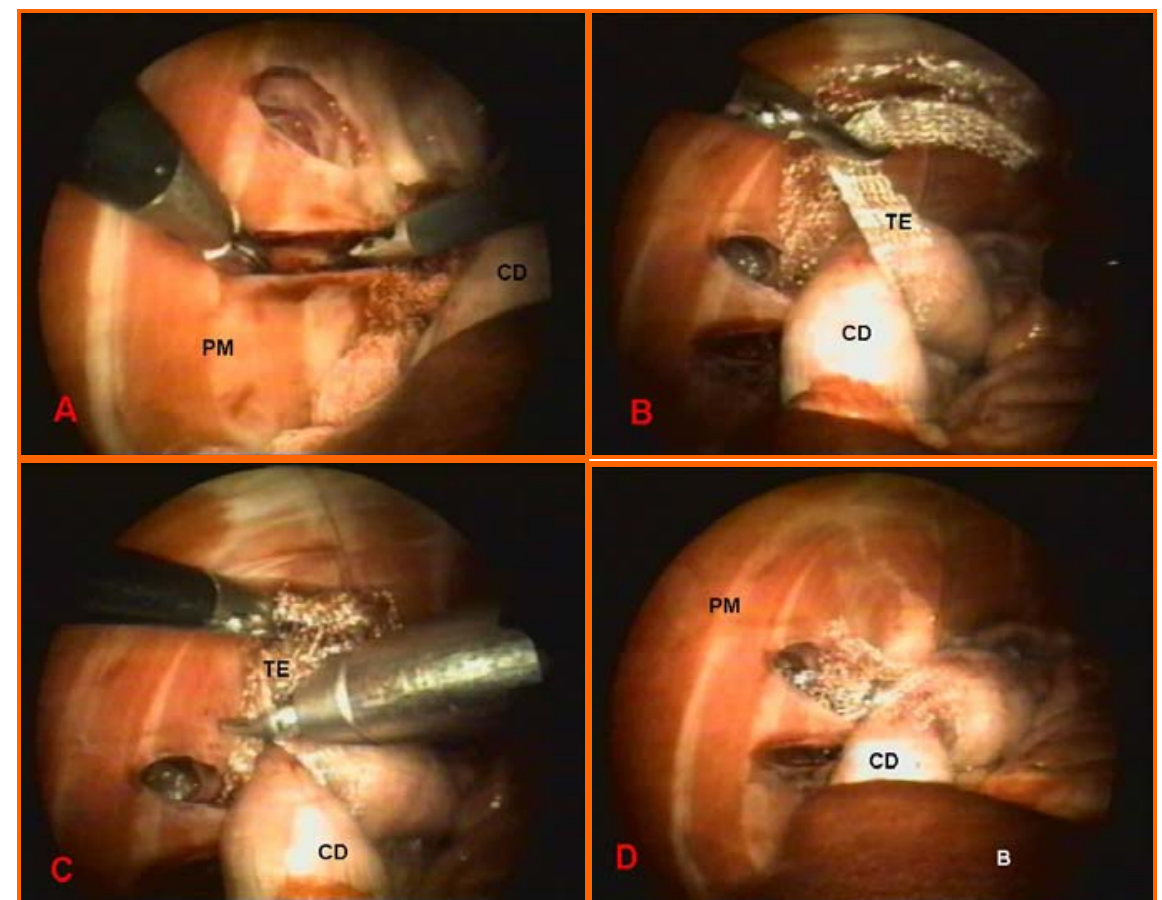

Figura 1. Técnica laparoscópica para colopexia com tela de polipropileno em cães. A) Realizam-se duas incisões transversais e paralelas entre si no músculo transverso abdominal esquerdo, produzindo flap bipediculado. B) Um segmento de tela de polipropileno é passado ao redor do cólon descendente, através de lesão produzida no mesocólon, e sobre o flap de músculo transverso abdominal. C) As extremidades da tela de polipropileno são unidas a partir da aplicação de clipe de titânio. D) Aspecto final da colopexia. O cólon descendente é mantido em contato com a parede muscular esquerda. $\mathrm{PM}=$ parede muscular; $\mathrm{CD}=$ cólon descendente; $\mathrm{TE}=$ tela de polipropileno; $\mathrm{B}=$ baço.
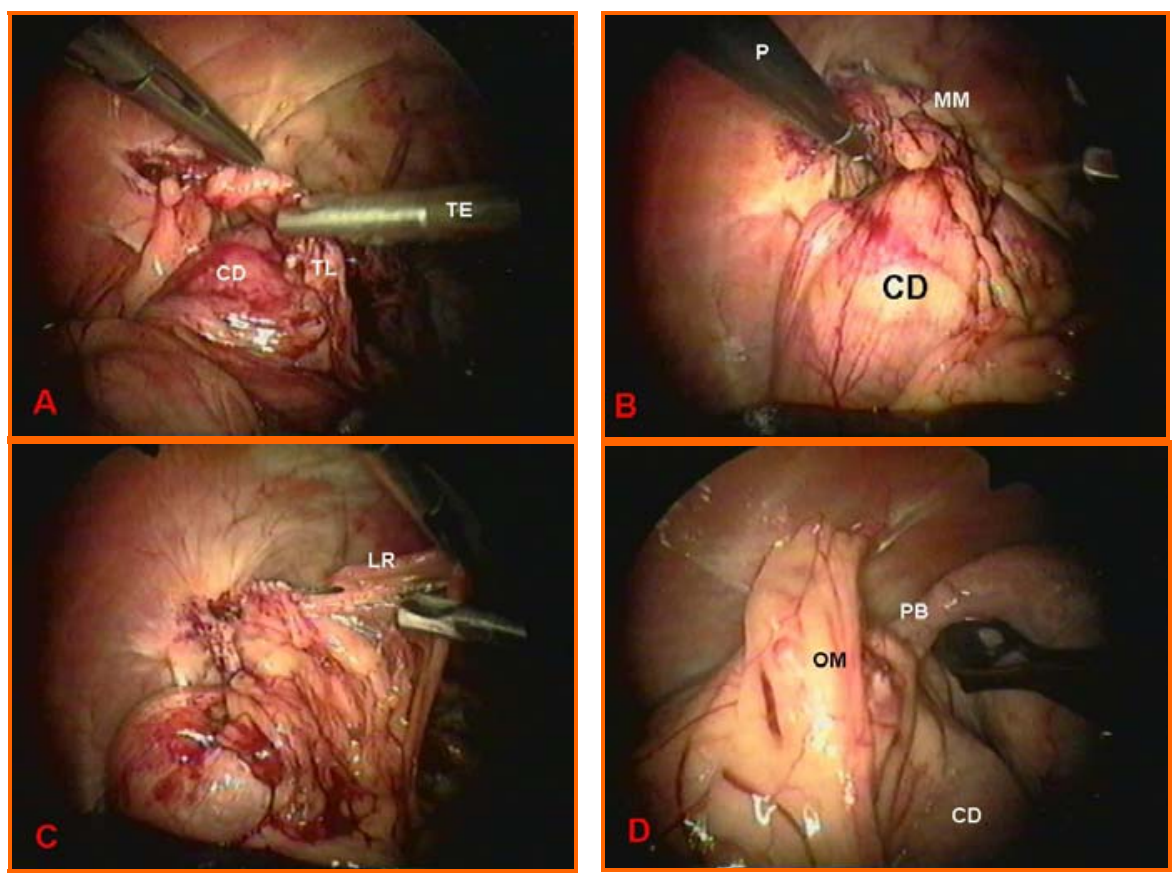

Figura 2. Observações macroscópicas pós-operatórias associadas às colopexias laparoscópicas com tela de polipropileno. A) A extremidade da tesoura laparoscópica (TE) aponta para tela de polipropileno (TL) que se encontra envolvida por tecido conjuntivo do paciente. O cólon descendente $(\mathrm{CD})$ apresenta-se aderido à tela que está fixada à parede muscular. B) Aderência do mesométrio (MM) junto à região de colopexia. A extremidade da pinça (P) está posicionada na lacuna existente entre o implante e o cólon descendente. C) Visibilização do ligamento redondo do útero (LR), que estava aderido à colopexia, após a sua secção (seta). D) Aderência do omento (OM) e da parede vesical $(\mathrm{PB})$ na região de colopexia. 
O paciente que apresentou o cólon completamente envolto pela tela (que estava fixada à parede muscular) sem aderência à mesma demonstrava deslocamento cranial ou caudal do órgão sem nenhum tipo de obstáculo. Essa condição possivelmente ocorreu devido à produção de lesão no mesocólon de dimensão maior que a necessária para acomodar adequadamente o implante. Associada a essa situação, a ocorrência de movimentos peristálticos poderia ter contribuído para a não formação de aderências entre a superfície medial do cólon e o implante. Em um outro estudo, descreve-se a presença de lesão permanente no mesocólon após a passagem de membrana conservada para a produção de colopexia nos moldes do presente trabalho, sendo que seus autores afirmaram que tal situação deve ser evitada pelo risco de herniação de estruturas. A partir do achado, os autores do presente trabalho passam a indicar a produção de lesão na superfície antimesentérica que permita ajustada acomodação do implante, além da aplicação de uma sutura intracorpórea (podendo a mesma ser realizada em padrão interrompido simples), abrangendo as camadas serosa e muscular da superfície medial do cólon em conjunto com o implante.

Um aspecto interessante quanto ao procedimento proposto recai sobre a sua aplicabilidade em animais de pequeno porte, já que a média de massa corporal dos pacientes foi menor que a $6,5 \mathrm{~kg}$. Ressalta-se que o prolapso retal recidivante, principal indicação cirúrgica para colopexia, freqüentemente ocorre em animais jovens com diarréia e tenesmo graves (Tobias, 1996), que, na maioria dos casos, são de pequeno porte. Em cavidades abdominais de pequenas dimensões, o espaço de trabalho obtido a partir do pneumoperitônio é restrito, o que torna a aplicação de suturas intracorpóreas dificultosa e, por conseqüência, mais demorada. Assim, a operação com o uso de tela poderá se tornar uma alternativa em relação à colopexia incisional laparoscópica para esses pacientes. Também, como possível vantagem em relação à cirurgia incisional, pode-se colocar a ausência de riscos de perfuração da mucosa do cólon com conseqüente contaminação (Popovitch et al., 1994; Fossum et al., 2002), quer seja pela aplicação inadvertida da agulha de sutura ou pela lesão não intencional dessa camada durante a incisão antimesentérica.
Ressalta-se a necessidade de utilização de materiais relativamente onerosos na operação experimentada, incluindo o segmento de tela de polipropileno e a aplicação de um a dois clipes, conforme a realização das alterações técnicas propostas nesse item. Contudo, se for considerado que uma única tela pode ser aplicada em vários procedimentos, haja vista a diminuta largura necessária para a implantação e a possibilidade de reesterilização do material, o valor de investimento se torna viável. Comparada com o procedimento incisional laparoscópico (Brun et al., 2004d, g), a técnica proposta necessita menor quantidade de material de sutura, menor consumo da lâmpada da fonte de luz e, possivelmente, menor consumo de $\mathrm{CO}_{2}$ medicinal.

Havia a preocupação quanto à ocorrência de tenesmo pós-operatório, megacólon e impactação fecal, já que o implante envolveria o cólon e poderia restringir sua capacidade de dilatação. Temia-se ainda que essa condição se agravasse graças à retração do tecido cicatricial que envolveria o implante e o intestino, bem como da ferida provocada. A manutenção de espaço entre a superfície antimesentérica do órgão e a tela, a partir da passagem de uma pinça através destes, demonstrou ser manobra efetiva, já que as possíveis complicações supracitadas não foram evidenciadas em nenhum dos pacientes. A esperada contração cicatricial (Coda et al., 2003) não esteve associada com diminuição importante do espaço em questão, possivelmente pela natureza pouco elástica do material.

Quanto às aderências intracavitárias, esperava-se observar a fixação do omento junto ao sítio de colopexia, haja vista que essa condição tem sido descrita em pacientes submetidos a procedimentos incisionais laparoscópicos e convencionais (Wilson et al., 1996; Rawlings, 2002) e que, no presente caso, haveria a permanência de um corpo estranho nessa região. Nas situações em que a tela fica exposta na cavidade peritoneal, é comum ocorrer aderências dessa natureza (Borrazzo et al., 2004). Deve-se também considerar que o omento pode se fixar às áreas sem peritônios em animais não submetidos à cirurgia (Henderson, 1982) e que essa condição geralmente não está associada a quaisquer alterações clínicas (Brun, 2004d, g). Outras fixações envolveram os ligamentos uterinos e o mediano da bexiga, situação previamente relatada em colopexias (Brun et al., 2004d) e que podem ser atribuídas à proximidade dos tecidos em questão. 
A presença de fixação da parede da bexiga observada em um dos pacientes, mesmo sem estar relacionada a alterações clínicas, torna-se preocupante, pois poderia desencadear alterações pós-operatórias na micção. Ainda, considerandose a ocorrência dessa alteração em animal nãocastrado, poderia haver risco de desordens gestacionais e/ou do parto, uma vez que o útero estaria localizado entre a bexiga e o cólon, e que ambas as estruturas estariam fixadas. Independentemente da presença de aderências vesicais, os presentes autores indicam a necessidade de ovário-histerectomia em animais submetidos à colopexia. Possivelmente, na cadela em que ocorreu essa complicação, a colopexia tenha sido realizada caudalmente em relação às demais, o que facilitaria o contato da bexiga com a tela, uma vez que essa condição não tem sido relatada nas diferentes técnicas de colopexia por celiotomia ou laparoscopia (Popovitch et al., 1994; Brun et al., 2004d, g). Para evitá-la, os autores do presente trabalho sugerem que se estabeleça a região de abrangência da bexiga quando repleta e que se promovam as incisões no músculo transverso abdominal cranialmente a esse limite.

As observações histológicas seguem o padrão esperado quando do uso de tela de polipropileno (Coda et al., 2003; Papadimitriou e Petros, 2005), já que esse implante é considerado um corpo estranho, condição que explica a presença de quantidade moderada à elevada de células mononucleares, mesmo em períodos relativamente longos do pós-operatório (30 a 42 dias). A permanência de tecido fibroso em elevada quantidade pode ser considerada como aspecto positivo, uma vez que a produção de colágeno está diretamente relacionada à resistência da ferida (Ellison, 1989). Possivelmente, esse tecido permitiu a formação de colopexia resistente, hipótese que poderia ser comprovada caso fossem realizados ensaios envolvendo aferições de resistência à tração, conforme previamente descrito em outros experimentos (Brun et al., 2004d; Rawlings et al., 2001; Wilson et al., 1996).

\section{CONCLUSÕES}

A técnica de colopexia laparoscópica com segmento de tela de polipropileno é viável para cães. Contudo, indicam-se pequenos ajustes para evitar o deslocamento da tela ou não formação de aderências, que incluem a aplicação de um clipe adicional unindo as extremidades do implante, e a confecção de sutura abrangendo este e as camadas serosa e muscular do cólon.

\section{AGRADECIMENTOS}

Aos colaboradores: Henrique Rolla Gonçalves, Guilherme Thomazzi, Wagner Rugeri, Júlio David Spagnolo, Michele Westephal de Ataíde, Janiele Cansi, Bruna Sgorla, Ricardo Zanella.

\section{REFERÊNCIAS BIBLIOGRÁFICAS}

BORAZZO, E.C.; BELMONT, M.F.; BOFFA, D. et al. Effect of prosthetic material on adhesion formation after laparoscopic ventral hernia repair in a porcine model. Hernia, v.8, p.108-112, 2004.

BRUN, M.V.; BARCELLOS, H.H.A.; GUIMARÃES, L.D. et al. Técnica proposta para colopexia laparoscópica em cão com retalho de pericárdio homólogo conservado em solução hipersaturada de sal a 75\%. In: CONGRESSO ESTADUAL DE MEDICINA VETERINÁRIA, 16.; CONGRESSO DE MEDICINA VETERINÁRIA DO CONESUL, 5.; 2004, Passo Fundo. Anais.... Porto Alegre, 2004a. (Resumo).

BRUN, M.V.; BARCELLOS, H.H.A.; OLIVEIRA, S.T. et al. Colopexia incisional laparoscópica no tratamento de prolapso retal recidivante em dois cães. Braz. J. Vet. Res. Anim. Sci., v.41, p.139-140, 2004b.

BRUN, M.V.; PIPPI, N.L.; BECK, C.A.C. et al. Colopexia incisional por celiotomia ou transparietal auxiliada por laparoscopia em cães. Cien. Rural, v.34, p.829-837, 2004c.

BRUN, M.V.; PIPPI, N.L.; BECK, C.A.C. et al. Resistência à tração de colopexias incisionais realizadas por cirurgia laparoscópica ou celiotomia em cães Ciên. Rural, v.34, p.839-845, 2004d.

BRUN, M.V.; GUIMARÃES, L.D.; BARCELLOS, H.H.A. et al. Colopexia incisional laparoscópica no manejo de prolapso retal recidivante em um cão. In: CONGRESSO BRASILEIRO DE VIDEOCIRURGIA VETERINÁRIA, 1., 2004, Porto Alegre. Anais... Porto Alegre, 2004e. p.23. (Resumo). 
BRUN, M.V.; GUIMARÃES, L.D.; BARCELLOS, H.H.A. et al. Colopexia laparoscópica no tratamento de prolapso retal recidivante em um cão submetido à colopexia por celiotomia. In: CONGRESSO BRASILEIRO DE VIDEOCIRURGIA VETERINÁRIA, 1., 2004, Porto Alegre. Anais... Porto Alegre, $2004 f$. p.25. (Resumo).

BRUN, M.V.; PIPPI, N.L.; BECK, C.A.C. et al. Avaliação de dois diferentes fios de sutura para colopexia incisional laparoscópica em cães estudo experimental. Braz. J. Vet. Res. Anim. Sci., v.41, p.154-161, 2004g.

CODA, A.; BENDAVID, R.; BOTTO-MICA, F. et al. Structural alterations of prosthetic meshes in humans. Hernia, v.7, p.29-34, 2003.

ELLISON, G.W. Wound healing in the gastrointestinal tract. Sem. Vet. Med. Surg. (Small Anim.), v.4, p.287-293, 1989.

FOSSUM, T.H.; HEDLUND, C.S.; HULSE, D.A. et al. Cirurgia do sistema digestório. In: Cirurgia de pequenos animais. São Paulo: Roca, 2002. p.222-405.

FREEMAN, L.J. Laparoscopic colorectal surgery. In: Veterinary endosurgery. St. Louis: Mosby, 1998. p.144-151.
PAPADIMITRIOU, J.; PETROS, P. Histological studies of monofilament and multifilament polypropylene mesh implants demonstrate equivalent penetration of macrophages between fibrils. Hernia, v.9, p.75-78, 2005.

POPOVITCH, C.A.; HOLT, D.; BRIGHT, R. Colopexy as a treatment for rectal prolapse in dogs and cats: a retrospective study of 14 cases. Vet. Surg., v.23, p.115-118, 1994.

RAWLINGS, C.A.; FOUTZ, T.L.; MAHAFFEY, M.B. et al. A rapid and strong laparoscopic-assisted gastropexy in dogs. Am. $J$. Vet. Res., v.62, p.871-875, 2001.

RAWLINGS, C.A.; HOWERTH, E.W.; MAHAFFEY, M.B. Laparoscopic-assisted cystopexy in dogs. Am. J. Vet. Res., v.63, p.1226-1231, 2003.

THOMPSON, S.E.; HENDRICKSON, D.A. Hernia repair. In: FREEMAN, L.J. Veterinary endosurgery. St. Louis: Mosby, 1998. p.105-112.

TOBIAS, K.S. Alimentary system. In: HARARI, J. Small animal surgery. Baltimore: Wiliams \& Wilkins, 1996. p.110-142 .

WILSON, E.R.; HENDERSON, R.A.; MONTGOMERY, R.D. A comparison of laparoscopic and belt-loop gastropexy in dogs. Vet. Surg., v.25, p.221-227, 1996. 